\title{
Architecture Design of Datacenter for Cloud English Education Platform
}

\author{
https://doi.org/10.3991/ijet.v14i01.9464 \\ Ming Chang, Min Zhang ( $\left.{ }^{\bowtie}\right)$ \\ Beihua University, Jilin, China \\ Mariazhang97@126.com
}

\begin{abstract}
Considering the importance of datacenter to cloud English education platform (CCEP), this paper designs a novel datacenter for the CEEP, which realizes resource pooling on the storage, network, computation and other infrastructures through integration of virtualization technology. Meanwhile, the intelligent, automatic and centralized service orchestration and scheduling management were implemented via the global CEEP system. On this basis, a complete datacenter solution was developed for the CEEP. Through performance analysis, it is concluded that the solution can significantly improve the effectiveness, resource utilization, service deployment efficiency as well as operation and maintenance management.
\end{abstract}

Keywords - Cloud English Education Platform (CEEP), datacenter, conceptual design

\section{Introduction}

The sharing of education resources is the key to the current English education reform in China. Based on cloud computing and virtualization, the cloud English education platform (CEEP), has become a popular tool for resource-sharing English education in the market and in universities. This novel education system is conducive to the informatization of English education and the development of English education business [1].

The operation of the CEEP relies on the supporting hardware, i.e. the datacenter. However, the datacenter construction now faces four major challenges, namely, the high cost of hardware, the low resource utilization, the complex configuration, and the difficulty in deployment. For hardware cost, the traditional datacenters require the purchase of multiple hardware and physical devices in advance, which pushes up the pre-work cost and drags down the yield in the initial phase. For resource utilization, the underlying resources of the traditional datacenter cannot be used flexibly; what is worse, resource borrowing and sharing are not allowed between different devices. For configuration, largescale cloud computing datacenters need massive server clusters and require tens of thousands of physical network devices to access and integrate virtual switches [2]. For business deployment, it is impossible to deploy traditional datacenters rapidly or move the related services in a flexible manner. 
These defects of the traditional datacenters, coupled with poor scalability, serious restrict the informatization of the CEEP. This calls for a new type of low-cost datacenters that support virtualization, intelligence, and easy expansion, and facilitate the allocation of available resources on demand.

In light of the above, this paper designs a novel datacenter for the CEEP, which realizes resource pooling on the storage, network, computation and other infrastructures through integration of virtualization technology. Meanwhile, the intelligent, automatic and centralized service orchestration and scheduling management were implemented via the global CEEP system. On this basis, a complete datacenter solution was developed for the CEEP. Through performance analysis, it is concluded that the solution can significantly improve the effectiveness, resource utilization, service deployment efficiency as well as operation and maintenance management.

The remainder of this paper is organized as follows: Section 2 reviews the relevant studies on the construction of the CEEP; Section 3 puts forward the datacenter design for the CEEP; Section 4 carries out the performance analysis of the proposed solution; Section 5 wraps up this paper with some meaningful conclusions.

\section{$2 \quad$ Literature Review}

Much research has been done at home and aboard on the construction of the CEEP. For example, Reference [3] introduces the concept of English education cloud by applying cloud computing technology in English education, explains the framework and functions of three English education cloud services centered on virtualization, and proposes the method for the construction of the basic CEEP. Reference [4] builds a new IT infrastructure platform, which provides users with stable and efficient access, improves hardware resource utilization and reduces maintenance and management costs.

Reference [5] builds a free, flexible and multi-purpose English education platform called Seattle, which combines cloud computing, grid computing, peer-to-peer network and distributed systems into an organic whole; multiple operation systems and architecture systems can be integrated on this platform to provide available computing resources for multi-user social platforms, eliminating the needs for dedicated infrastructure. Reference [6] identifies the most common infrastructure of cloud computing architecture for e learning through extensive review, and verifies the applicability of the infrastructure to the data mining of English education using some examples of cloud computing-based e learning.

Reference [7] underlines the importance of cloud computing in English education, and ascertains the most common cloud service environment in universities, noting that cloud computing enables users to access data and software anywhere, as long as there is Internet access. Reference [8] explores the technology acceptance and application based on cloud computing e-learning system, and carries out a survey on multiple participants in e-learning based on cloud computing and an analysis using a structural equation model, revealing that e-learning based on cloud computing is influenced by performance expectations, social impact and hedonic motivation. 
Reference [9] designs a global open online learning system, capable of storing mass data and integrating vast learning resources, based on Google's cloud computing platform and Google App Engine, aiming to improve the integration of online learning information resources and encourage teacher-student interaction. Reference [10] probes into cloud computing and its application in big data processing of higher distance English education, and presents a parallel k-means clustering algorithm based on cloud computing platform Hadoop [11].

Based on the previous studies, this paper investigates the application and construction of the CEEP in the following steps: the conceptual design of the datacenter for the CEEP was carried out, and a datacenter architecture was then built to integrate the cloud network based on computation/storage/network virtualization technologies. The proposed solution enables global service orchestration and deployment with the CEEP and network controller, laying the basis for coordinated scheduling for global English education resources.

\section{Conceptual Design for CEEP Datacenter}

As its name suggests, the CEEP is implemented based on cloud computing. Through co-building and sharing of resources, the platform provides users with a comprehensive, high-quality solution for English education, and achieves centralized management by high-speed network, high-performance computing, cloud computing and other key technologies. Here, the design concept of the proposed CEEP datacenter is elaborated from the angles of system integration, network architecture, computation architecture and storage architecture.

\subsection{System Integration}

The overall architecture of the datacenter was inspired by software definition. Through scenario- and module-based design, the physical architecture was decoupled from the infrastructure, and the infrastructure was decoupled from the service modules. In this way, new business could go alive quickly thanks to the scalability of the datacenter and the dynamic expansion of services.

The authors created a software-defined infrastructure based on a universal hardware design with typical specifications, with the aid of virtualization technology. The concise and reliable infrastructure is easy to deploy, manage, expand and upgrade, allowing the management, supervision, analysis and virtualization of computing, storage, and network and other resources.

The resource pool in the infrastructure guarantees the scientific management of all physical resources. As a result, the users are provided with better investment protection, and a variety of datacenter requirements are satisfied, including but not limited to new construction, upgrading, expansion, as well as global management and control.

Since the datacenter supports computing, network and storage in English education system, it was integrated with key technologies and development trends in English education market, forming a module-resource pool management platform capable of 
scientific management of the datacenter. As mature datacenter management software, the resource pool management platform facilitates the global deployment, allocation and supervision of underlying IT resources, changes the scale of resource allocation dynamically on demand and adapts to the dilatation of different applications quickly

The overall architecture of the datacenter is illustrated in Fig. 1 below.

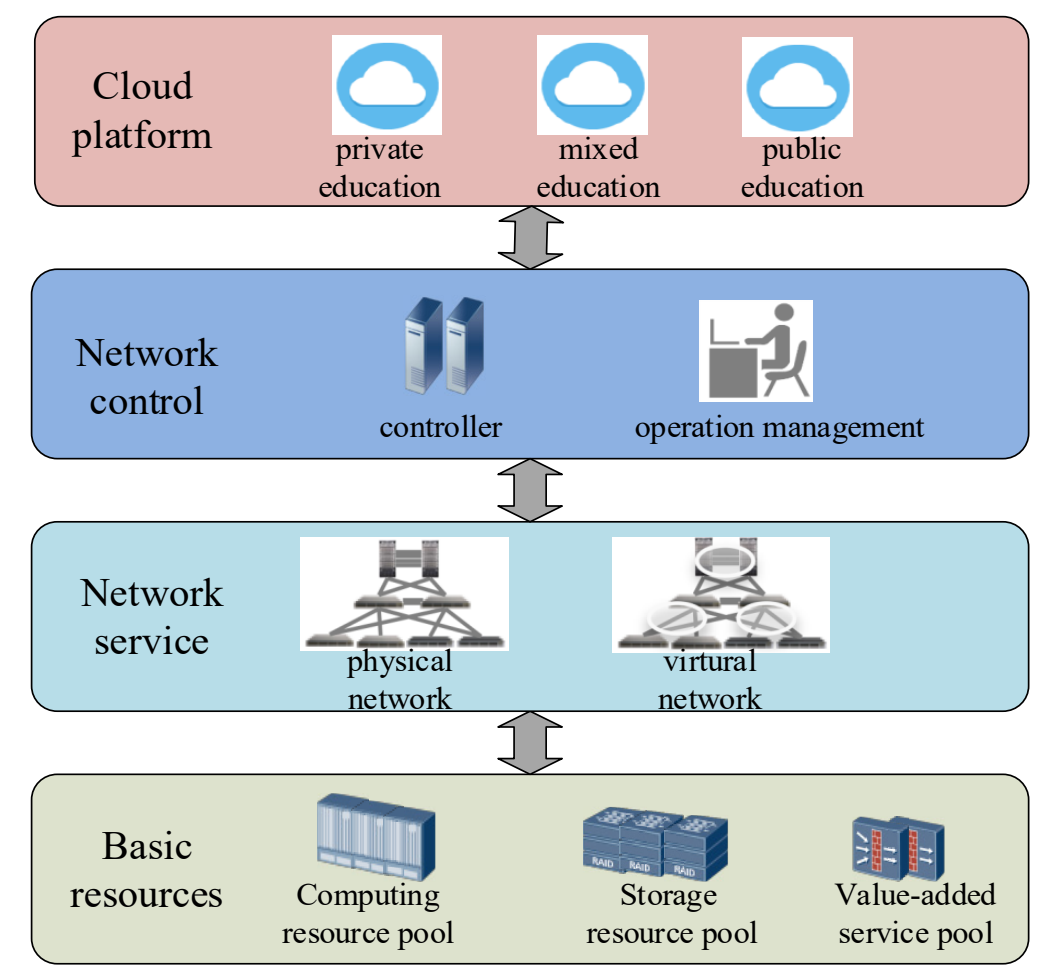

Fig. 1. Overall architecture of datacenter solution

\subsection{Network Architecture}

In traditional datacenters, the network architecture consists of such three levels as the core level, the convergence level and the access level. The three-layer structure leads to many problems, e.g. network congestion and low data conversion efficiency.

In view of these, the proposed datacenter adopts a flat core network architecture based on the SDN. Involving data and control planes, the network architecture achieves the intelligent management on the underlying equipment for physical network, and provides a software-defined virtualization network for the upper layer application. As shown in Figure 2, the virtualization component at the core of the network architecture provides an expansion mechanism for the system, and enables the global control over network resources, including hardware devices (e.g. switches, routers, firewalls, and load balancers) and devices in the virtual environment. 
This network architecture enjoys the following advantages. The hardware cost is low thanks to the network switching based on universal devices and software-defined methods. The network is fully utilized due to the application of cloud computing in dynamic scheduling of resource pool. The network is simple and high performing, allowing the traffics for high-performance server. The operation and maintenance costs are reduced with the provision of a global network management interface. The network is highly reliable as link loop problems and network downtime are solved by virtual cluster and stacking technology.

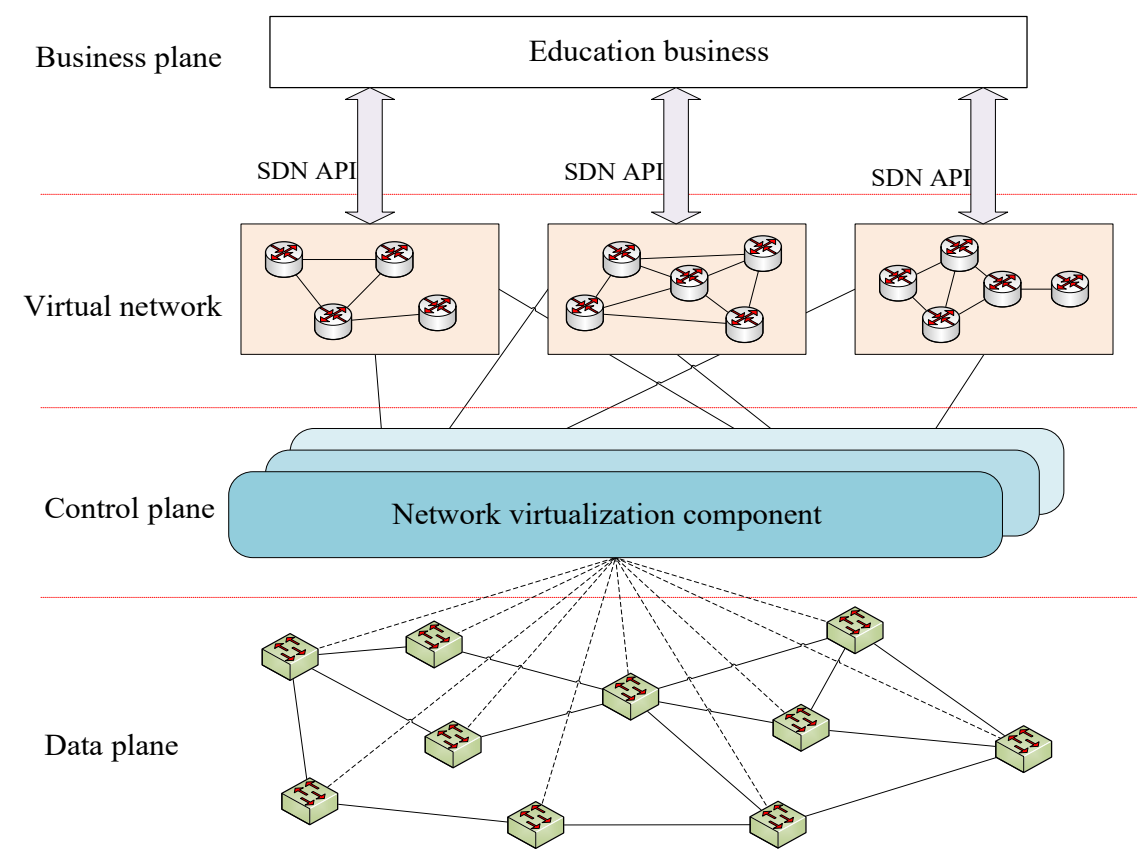

Fig. 2. Network architecture

\subsection{Computation Architecture}

With the aid of the virtualization technology, the datacenter integrates the computing resources from the underlying physical devices, and satisfies the users' demand for flexible and reliable computing capacity. As shown in Figure 3, the virtualization service architecture encompasses an access level, a scheduling level, a driver level, and a virtualization level.

Specifically, the access layer receives the network request via a standard interface. The scheduling layer selects the appropriate driver in light of the policy configurations, and sends the request information to the driver for treatment. The driver layer, as the core of the virtualization subsystem, converts the request information into a format that the virtualization layer can process via each virtualization software driver. Besides, this layer runs the appropriate virtualization software, and performs resource 
operations. The virtualization layer realizes global management on heterogeneous virtualization resources for the system (e.g. VMware, KVM and Docker).

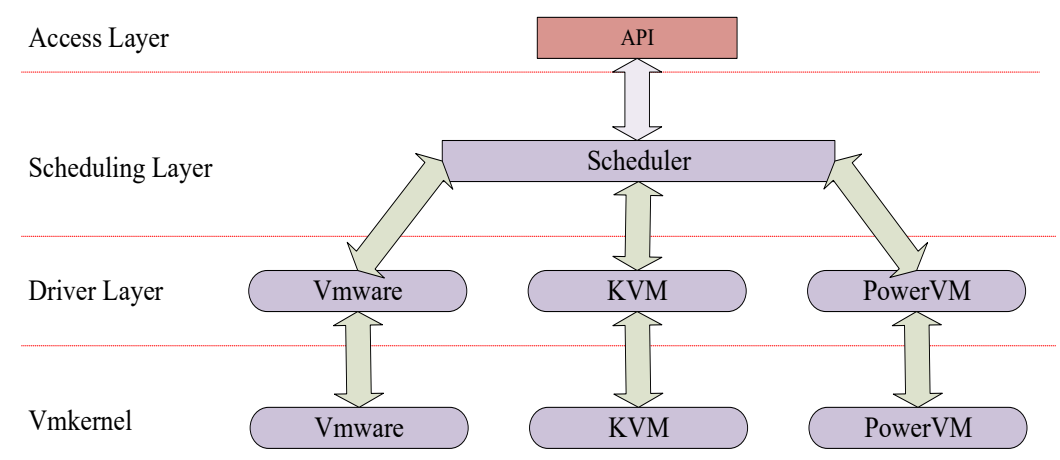

Fig. 3. Virtualization service architecture

The main functions of the virtual machine subsystem range from resource synchronization, virtual machine management, host management, to performance acquisition. Resource synchronization refers to synchronizing data between the underlying environment and resource pool, as well as between resource pool and virtual machine. Virtual machine management stands for the creation, startup/shutdown and change of virtual machine. Host management covers cluster management, storage management, and port group management, among which, cluster management ensures load balancing and high availability in the cluster. Performance acquisition means the collection of performance data (e.g. as CPU and memory) of physical and virtual machines in the current environment to supervise the underlying resources.

\subsection{Storage Architecture}

The datacenter adopts software-defined storage (SDS) to facilitate the pooling of all resources and create a friendly user interface and improved API. In this way, the users can perform scale-up or scale-out without any more workloads. The SDS makes it possible to deploy and manage heterogeneous or proprietary storage platforms, featured by automation, standard interfaces, virtual data paths, scalability and transparency.

The SDS includes three functions, namely, storage management, data service and data request service. The storage management refers to the building of a storage resource pool through the depiction and virtualization of the features for storage resources like flash disk, mechanical disk, storage array, and JBOD from the server via storage management protocol (such as SMI-S). After resource pooling, the data services like space deployment, data availability, data protection, and data security are provided according to the user's storage service level. The data request service includes responding to the software developer's data request via storage resource invoke interface, and providing the appropriate storage resource to the user according to his/her service level. 


\section{$4 \quad$ Performance Analysis}

The performance of the proposed datacenter design was discussed from the following perspectives: deployment automation, operation and maintenance visualization, and access flexibility.

\subsection{Deployment automation}

As shown in Fig. 4, the CEEP platform, working with the network controller, transfers the application, logic, and physical networks for end-to-end automatic deployment, and supports minute-level service online. Specifically, the cloud management platform converts the application view into a logic network view based on the service perspective, and defines a dedicated network for that view. Next, network controller converts the logical network into a physical network according to the physical network resources, and automatically delivers the configurations.

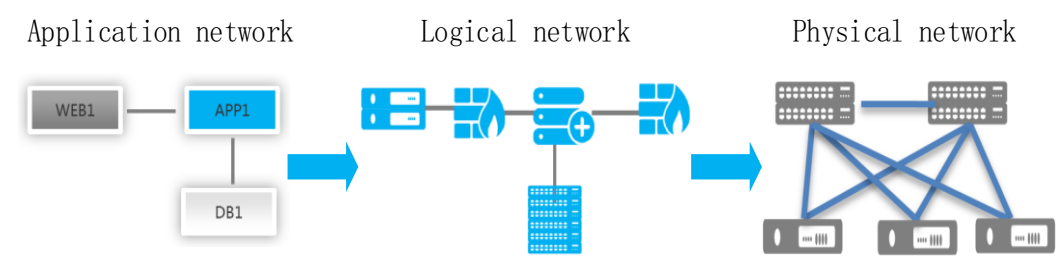

Fig. 4. Conversion among network views

\subsection{Operation and Maintenance Visualization}

As shown in Fig. 5, network status information is globally logged and analyzed through the visualization of network resources, services and quality. Based on big data, the intelligent analysis enables minute-level fault positioning, improves operation and maintenance efficiency, and reduces operation and maintenance costs. Thanks to network visualization, the network resources can be displayed, supervised, managed and utilized based on the traffic of specific application. Besides, the visualization of network services supports the conversion among the application, logic, and physical topologies. 


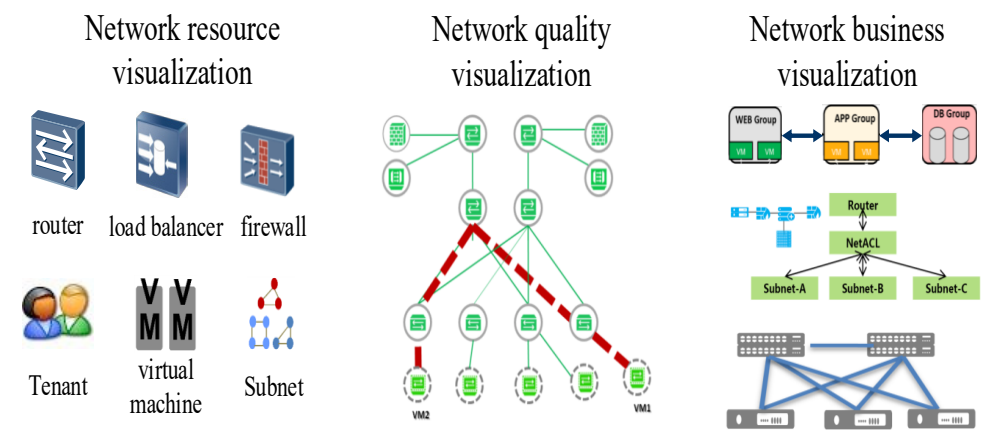

Fig. 5. Visualization of network operation and maintenance

\subsection{Access Flexibility}

As shown in Fig. 6, the virtualized network decouples the logical network from the physical network, supports dynamic migration of virtual machine, optimizes the transmission path, enables the sharing and flexible scheduling of computing and storage resources, and improves the use of network, storage, and computing resources.

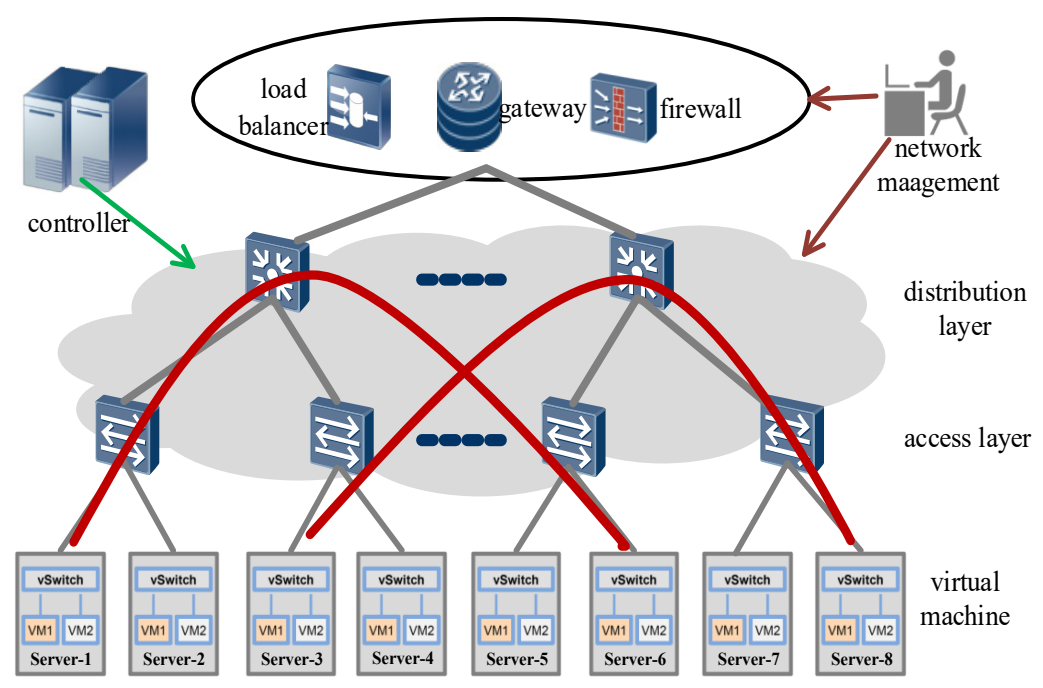

Fig. 6. Network resilient coupling

\subsection{Index Analysis}

The indices of the proposed datacenter architecture were theoretically analyzed and compared with those of the traditional architecture. According to the results in Table 1 , the proposed datacenter reduced the pre-construction cost by over $40 \%$ from the level of the traditional architecture, because it only deploys hardware devices required by actual services. Besides, the proposed architecture utilized more than $70 \%$ of the 
available resources using $45 \%$ less energy than the traditional architecture. This is because a global resource pool is formed in the IT systems and over $90 \%$ services run on the virtualization platform. In terms of service deployment efficiency, the proposed architecture reduced the business deployment time by $60 \%$, making it possible for rapid service expansion. Moreover, no data went missing, no downtime occurred, all faults were detected automatically and all services were migrated automatically in the proposed architecture, an evidence for seamless service protection. The $30 \%$ increase in operation and maintenance efficiency is attributable to the datacenter management software, which manages multiple resource pools and displays the full running view; the users are free to view system status at any time.

Table 1. Comparison between traditional and cloud platform programs

\begin{tabular}{|l|l|l|}
\hline \multicolumn{1}{|c|}{ Items } & Traditional architecture & \multicolumn{1}{c|}{$\begin{array}{c}\text { Proposed datacenter architec- } \\
\text { ture }\end{array}$} \\
\hline CPU utilization & $10 \%$ & $50 \%$ \\
\hline Memory utilization & $20 \%$ & $70 \%$ \\
\hline Network bandwidth utilization & $30 \%$ & $60 \%$ \\
\hline Unexpected downtime & Several hours & Zero \\
\hline System recovery time & $1 \mathrm{~h}$ & $5 \mathrm{~min}$ \\
\hline Business deployment time & $30 \mathrm{~min}$ & $10 \mathrm{~min}$ \\
\hline System consumption & High & Low \\
\hline Single point fault & Yes & No \\
\hline
\end{tabular}

\section{Conclusion}

In view of the importance of the CEEP in English education and its dependence on datacenter, this paper elaborates the design of datacenter for the CEEP from the perspectives of system integration, network architecture, computation architecture and storage architecture, and realizes resource pooling on the storage, network, computation and other infrastructures with the aid of virtualization technology. The proposed CEEP, working with network controller, supports global business choreography and scheduling management, and features automatic deployment, visualized operation and maintenance and flexible connection. Through performance analysis, it is learned that the proposed CEEP datacenter architecture performed well in total cost, resource utilization, service deployment efficiency and operation and maintenance.

\section{Acknowledgement}

This work is funded by the Important Teaching Project of Beihua University (Grant No.: XJZD2018023)

\section{$7 \quad$ Reference}

[1] Ercan, T. (2010). Effective use of cloud computing in educational institutions. Social and Behavioral Sciences, 2(2): 938-942. https://doi.org/10.1016/j.sbspro.2010.03.130 
[2] Bora, U., Ahmed, M. (2013). E-Learning using Cloud Computing. International Journal of Science and Modern Engineering, 1(2): 9-13.

[3] Zhang, M., Zhou, J. (2014). Construction of Basic Education Cloud Computing Platform Based on Virtualization Technology. International Conference on Intelligent Computing, 709-716.

[4] Fu, T., Yang, J., Gao, Y., Yu, G. (2014). Constructing Cloud Computing Infrastructure Platform of the Digital Library Base on Virtualization Technology, Computer Engineering and Networking. Lecture Notes in Electrical Engineering, 747-754. https://doi.org/10.1007/978-3-319-01766-2_86

[5] Cappos, J., Beschastnikh, I., Krishnamurthy, A., Anderson, T. (2011). Seattle: a platform for educational cloud computing. Acm Sigcse Bulletin, 41(1): 111-115. https://doi.org/10.1145/1539024.1508905

[6] Fernández, A., Peralta, D., Benítez, J.M. (2014). e-learning and educational data mining in cloud computing: an overview. International Journal of Learning Technology, 9(1): 25-52. https://doi.org/10.1504/IJLT.2014.062447

[7] Lim, N., Grönlund, A., Andersson, A. (2015). Cloud computing: The beliefs and perceptions of Swedish school principals. Computers \& Education, 84: 90-100. https://doi.org/10.1016/j.compedu.2015.01.009

[8] Nguyen, T.D., Nguyen, T.M., Pham, Q.T. (2014). Acceptance and use of e learning based on cloud computing: the role of consumer innovativeness. International Conference on Computational Science and Its Applications. Springer International Publishing, 8553(5): 159-174.

[9] Zhao, K., Yang, Q., Ma, X. (2017). Exploration of an open online learning platform based on google cloud computing. International Journal of Emerging Technologies in Learning, 12(7): 17. https://doi.org/10.3991/ijet.v12i07.7249

[10] Zhao, K., Yang, Q., Ma, X. (2017). Exploration of an open online learning platform based on google cloud computing. International Journal of Emerging Technologies in Learning, 12(7): 17. https://doi.org/10.3991/ijet.v12i07.7249

[11] Zhang, X. (2018). Supporting EFL learners' reflective practices through systemic functional linguistic praxis: Alongitudinal case study. Educational Sciences: Theory \& Practice, 18(2), 495-513.

\section{Authors}

Ming Chang, author, lecturer, vice dean of English department of Foreign Languages College of Beihua University. Chang has participated in online education and several other important projects of teaching innovation of the university, and published several books on English education.

Min Zhang, corresponding author, associate professor, excellent English teacher of Foreign Languages College of Beihua University. Zhang has published several articles in Chinese core journals, led several projects of teaching innovation, and written several books on English education.

Article submitted 26 June 2018. Resubmitted 17 July 2018. Final acceptance 15 October 2018. Final version published as submitted by the authors. 